\title{
Estudio de la posible variación de la velocidad de la luz en el vacío utilizando el modelo de los cuantos vectoriales
}

\author{
Oscar Monrroy ${ }^{* 1}$ y Marco Merma ${ }^{1}$ \\ ${ }^{1}$ Universidad Nacional Mayor de San Marcos, Facultad de Ciencias Físicas, Lima, Perú
}

Recibido 15 enero 2020 - Aceptado 19 marzo 2020

\begin{abstract}
Resumen
Se investiga la propagación de la luz en el 99,99\% de espacio vacío existente en los sistemas naturales a un nuevo nivel de descripción, asumiendo que la luz es la única influencia que puede transmitir información. Primero se considera el campo electromagnético como sistema de referencia no inercial y se deduce mediante un razonamiento geométrico que el vector de propagación de la onda electromagnética cambia de dirección en cada instante de tiempo debido a la velocidad angular asociada al plano de vibración del campo eléctrico/magnético. Como consecuencia, la trayectoria real de un rayo de luz tendría la forma de una espiral cónica. El análisis geométrico de la trayectoria de los rayos de luz en el vacío conduce a una formulación simple de las ecuaciones paramétricas que representarían la cinemática esencial de los puntos del espacio vacío de los sistemas naturales (el sustrato). Con ello se deduce una ecuación que describe la variación de la velocidad de la luz con el tiempo. Esta ecuación indica dos posibles direcciones opuestas para la velocidad de la luz en el vacío de los sistemas naturales. Introduciendo el modelo del cuanto vectorial local, el cual se define como la velocidad angular cuantificable a toda escala espacial que dirige a los rayos de luz, se demuestra que la velocidad de la luz en el espacio libre (común a los sentidos) sería constante con respecto a un sistema de referencia inercial hipotético, donde se prescinde del cuanto vectorial asociado al rayo de luz. Se muestra que el modelo del cuanto vectorial permite interpretar la doble dirección de la velocidad de la luz en términos de una simetría especular al considerar que si una de las direcciones del cuanto vectorial corresponde a la propagación de la luz en el espacio vacío real de los sistemas naturales, la otra dirección correspondería a la propagación de la luz en el espacio vacío virtual de los sistemas naturales. También, se muestra que el modelo del cuanto vectorial permite establecer una condición de cuasiequilibrio entre los cuantos vectoriales asociados al espacio vacío real y virtual, la cual explicaría la percepción de la propagación rectilínea de la luz en el espacio libre común a los sentidos.
\end{abstract}

Palabras clave: Cuanto vectorial local, radio de acción, espiral cónica, velocidad variable de la luz, condición de cuasiequilibrio de los cuantos vectoriales..

\section{Study of the possible variation of the light speed in a vacuum using the vector quanta model}

*omonrroyc@unmsm.edu.pe 


\begin{abstract}
The propagation of light in $99,99 \%$ of empty space existing in natural systems is investigated to a new level of description, assuming that light is the only influence that can transmit information. First, the electromagnetic field is considered as a non-inertial reference system and it is deduced by geometric reasoning that the propagation vector of the electromagnetic wave changes direction at each instant of time due to the angular velocity associated with the plane of vibration of the electric/magnetic field. Consequently, the actual path of a ray of light would have the shape of a conical spiral. The geometric analysis of the path of light rays in a vacuum lead to a simple formulation of the parametric equations that would represent the essential kinematics of the points of the empty space of natural systems (the substrate). This leads to an equation that describes the variation of the speed of light over time. This equation indicates two possible opposite directions for the speed of light in the vacuum of natural systems. By introducing the local vector quantum model, which is defined as the quantifiable angular velocity at all spatial scales that direct light rays, it is shown that the speed of light in free space (common to the senses) would be constant with respect to a hypothetical inertial reference system, where the vector quantum associated with the ray of light is ignored. It is shown that the vector quantum model allows the double direction of the speed of light to be interpreted in terms of specular symmetry, considering that, if one of the directions of the vector quantum corresponds to the propagation of light in the actual empty space of natural systems, the other direction would correspond to the propagation of light in the virtual space of natural systems. Also, it is shown that the vector quantum model allows establishing a quasi-equilibrium condition between the vectorial quanta associated with said actual and virtual spaces, which would explain the perception of the rectilinear propagation of light in the free space common to the senses.
\end{abstract}

Keywords: Local vectorial quantum, radius of action, conical spiral, variable speed of light, quasiequilibrium condition of vectorial quanta..

\section{Introducción}

Desde la época de los astrónomos Ole Roemer (16441710) y Christian Huygens (1629-1695) la medición de la rapidez de la luz en el espacio libre, común a los sentidos, condujo a la conclusión de que tiene un valor finito. El primer método exitoso para medir la rapidez de la luz fue realizado en 1849, por A.H.L. Fizeau utilizando técnicas terrestres. Posteriormente, las mediciones realizadas por otros investigadores dieron el valor aproximado de $300,000 \mathrm{~km} / \mathrm{s}$ [Ser06]. Pero, en todas las mediciones el escenario (el espacio libre perceptible) a través del cual se propaga la luz siempre se consideró implícitamente un sistema inercial, y la trayectoria de los rayos luminosos se describe por líneas rectas.

Pero si el universo es dinámico, no es razonable admitir la existencia de un sistema de referencia inercial ni siquiera en teoría [Mon18]. Además, Einstein e Infeld [Ein86], llegaron a la conclusión de que no hay modo de hallar un sistema de referencia inercial, y por consiguiente es una ficción. En consecuencia las leyes clásicas, como las del Electromagnetismo y de la Óptica, que describen la propagación de la luz carecen de un sistema de referencia en el cual sean válidas. Si no existe un sistema de referencia inercial, entonces cada punto del espacio libre perceptible no debe considerarse inmóvil o con movimiento de traslación uniforme. En consecuencia la trayectoria real de los rayos luminosos no sería rectilínea y la rapidez de la luz podría cambiar con el tiempo.
Históricamente el experimento más conocido para investigar cambios en la rapidez de la luz fue realizado en 1887 por A.A. Michelson y E.W. Morley. En aquella época el sistema de referencia universal era un éter hipotético, que ocupaba todo el espacio, y cuyo movimiento debería afectar la rapidez de la luz según se propague a favor o en contra de la corriente del éter. Los resultados del experimento de Michelson y Morley (la ausencia del diagrama de interferencia de la luz utilizada) significaba que no existía ningún sistema de referencia absoluto respecto al cual se pueda detectar variaciones de la rapidez de la luz [Haw05].

Durante casi un siglo, desde que se proclamó el postulado de la constancia de la velocidad de la luz en el vacío común, por la teoría de la relatividad especial de Einstein en 1905, se aceptó como un hecho irrefutable que la velocidad de la luz en el vacío común era una constante cosmológica a toda escala espacial. Sin embargo, se han observado dificultades que produce este postulado respecto a la elaboración de una teoría cuántica de la gravedad [Mag06].

También, durante las últimas dos décadas de este siglo se han desarrollado las llamadas teorías VLS (Variying Speed of Light), las cuales marcaron el inicio de una nueva etapa de investigación fundamental sobre la naturaleza de la luz [Meh08], [Mof02], [Pet88], [Rac09]. Pero, las contribuciones de estas teorías siempre han dependido, en mayor o menor grado, de las teorías físicas convencionales y los resultados no han trascendido signi- 
ficativamente.

En el presente trabajo se investiga la propagación de la luz en el espacio vacío existente en todos sistemas naturales a otro nivel de descripción de la Física, donde se considera que la luz es la única influencia que puede transmitir la información. En un artículo anterior [Mon18], dicho espacio vacío se asume como una superficie tridimensional, que es la frontera de una inimaginable esfera tetradimensional, cuyo centro se puede considerar como un sistema de referencia inercial en primera aproximación.

Por consiguiente, el espacio vacío de los sistemas naturales, descrito como la superficie tridimensional o frontera de la esfera tetradimensional, constituye el sustrato esencial donde la luz es la influencia que transmite información organizada para el funcionamiento inteligente y la evolución temporal de los sistemas naturales.

\section{La propagación rectilínea de la luz}

El estudio tradicional de la propagación de la luz, que se muestra en los libros de enseñanza, la descripción geométrica de la propagación de la luz en el espacio libre perceptible se realiza presuponiendo que es en línea recta [Hec77], [Ros66].

Considérese una fuente puntual de luz en reposo situada en el espacio libre en un punto $\mathrm{O}$, como muestra la Figura 1. Asumiendo que la fuente de luz se encuentra en reposo, los frentes de onda serán superficies esféricas concéntricas cuyos radios crecen con la rapidez constante $c=$ $3 \times 10^{8} \mathrm{~m} / \mathrm{s}$. Además, puesto que las ondas electromagnéticas son transversales, los vectores eléctrico $\vec{E}$ y magnético $\vec{B}$ son tangentes en cada punto de los frentes de onda y perpendiculares al vector de propagación de la onda $\vec{k}$.

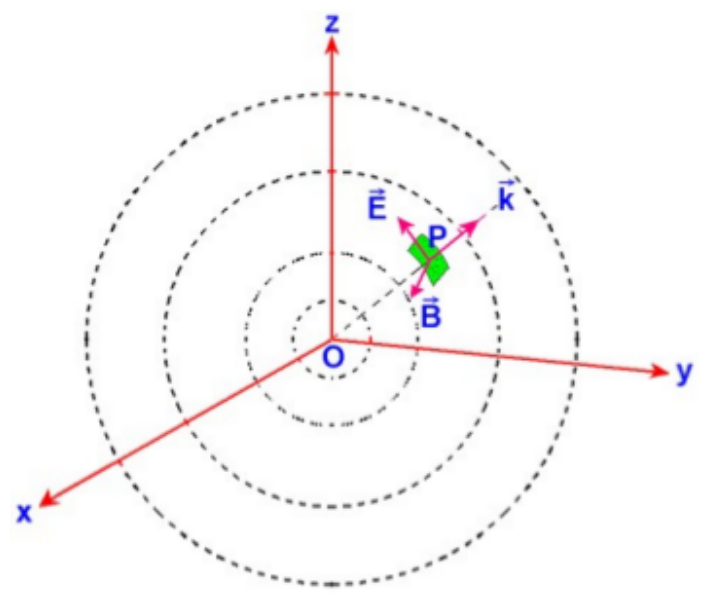

Figura 1: Frentes de onda esféricos procedentes de una fuente puntual de luz en reposo en $\mathrm{O}$. El vector de propagación se traslada rectilíneamente a lo largo de la línea resta OP
Es claro que en cada punto del frente de onda el vector de propagación $\vec{k}$ apunta en la dirección radial a medida que el frente de onda se expande desde la fuente inmóvil. Por ejemplo, a medida que el frente de onda se propaga desde el punto $\mathrm{O}$ al punto $\mathrm{P}$ el vector de propagación se traslada rectilíneamente hacia el exterior a lo largo de la línea $O P$. Debido a este comportamiento se dice que la luz viaja en línea recta en el espacio libre. La recta OP sobre la que se desplaza hacia fuera el vector $\vec{k}$ se llama rayo de luz. Puede imaginarse el patrón de rayos de luz emergiendo de la fuente puntual, tal como se muestra en la Figura 2. Estos rayos siempre serán perpendiculares a los frentes de onda esféricos.

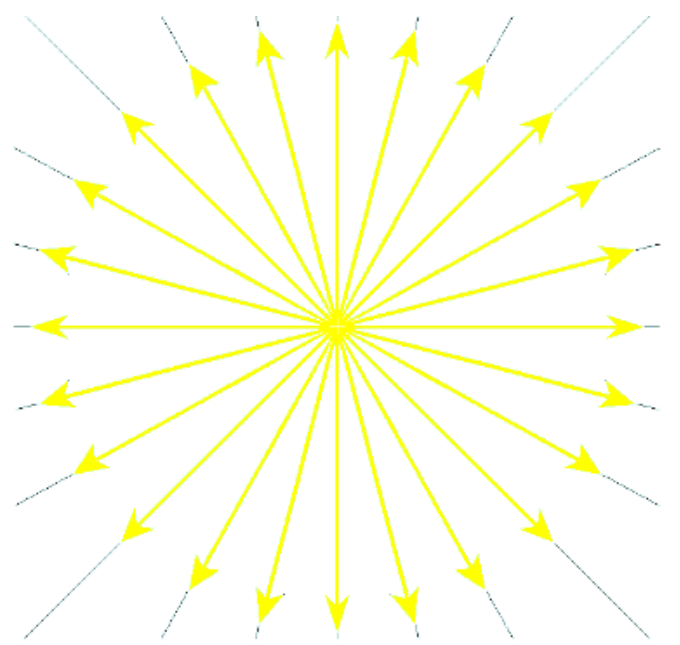

Figura 2: Patrón de rayos de luz que emergen de una fuente puntual inmóvil. Estos rayos siempre son perpendiculares a cualquier frente de onda esférico.

También, en el estudio tradicional de la propagación rectilínea de la luz se presenta un enigma: la polarización de la luz. Este fenómeno se estudia en términos de la dirección de oscilación de los vectores eléctrico y magnético. Cuando decimos que la luz natural no está polarizada significa que los vectores eléctrico y magnético vibran en todas las direcciones posibles. Si todas las direcciones de vibración son eliminadas, excepto una dirección particular, se dice que la luz está polarizada. Esto se consigue interponiendo en el camino de la luz un filtro polarizador con lo cual la luz emerge con una sola dirección de vibración. A su vez esta dirección de vibración define el eje de polarización del filtro.

Imagínese un experimento que consiste en dos filtros polarizadores orientados de tal modo que impiden totalmente el paso de la luz. Si colocamos un tercer filtro paralelo a los otros dos, ya sea delante o detrás de ellos, la luz no puede pasar y se ve oscuro. Pero si colocamos el tercer filtro entre los dos primeros de tal modo que su eje de polarización tenga una dirección diferente respecto a los ejes de los otros dos filtros, entonces según el sen- 
tido común es de esperar que siga viéndose oscuro. Sin embargo, se observa que la luz puede pasar.

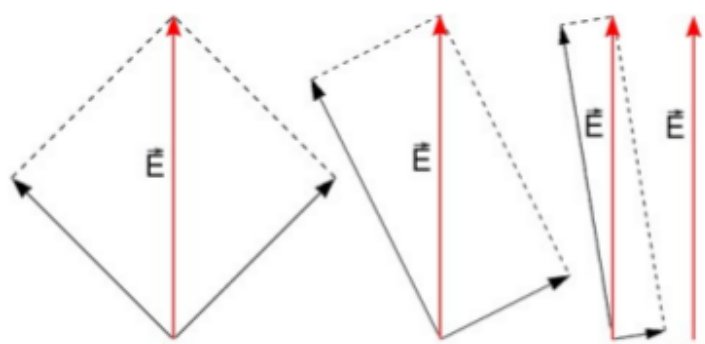

Figura 3: Descomposición del vector eléctrico de una onda electromagnética polarizada. El número de pares de componentes diferentes es infinito, porque cambian de dirección en cada instante. Pero, ¿qué es lo que está girando realmente?

¿Cómo se explica este hecho? Puesto que la luz tiene naturaleza vectorial, la explicación se puede realizar mediante la descomposición del vector eléctrico. Para una dirección dada del vector eléctrico existirán infinitos pares de componentes perpendiculares entre sí. En la Figura 3 se muestran algunos componentes del vector eléctrico. Los componentes que tengan la dirección del eje de polarización lograrán pasar, pero esto significará qué la luz nunca sería bloqueada completamente, lo cual está en contradicción con el experimento descrito en el párrafo anterior. Además, obsérvese que los componentes del vector eléctrico están girando, cambiando de dirección a cada instante debido a la descomposición continua del vector eléctrico. Pero, ¿qué es lo que está girando realmente? Más adelante se intentará descifrar este misterio [Mon19].

\section{Descripción en un sistema no inercial}

Considérese el siguiente experimento: imagínese que un rayo de luz natural (no polarizada) se dirige directamente hacia el lector saliendo perpendicularmente del plano del papel. El vector campo eléctrico de dicho rayo vibra en todas las direcciones posibles. Estas vibraciones están representadas por las flechas dobles (Figura 4a). Si interponemos en el camino del rayo un filtro polarizador cuyo eje de polarización está orientado horizontalmente, sólo los componentes horizontales de cada vector eléctrico pueden atravesar el filtro y la luz que emerge estará polarizada linealmente en la dirección horizontal (ver Figura 4b).

Si el vector campo eléctrico oscilara armónicamente en un plano fijo, como indica la doble flecha en la Figura 5, entonces cada punto del espacio (que constituye el sustrato) tendría movimiento armónico simple. Puesto que este movimiento es un componente del movimiento circular uniforme, entonces cada punto del espacio libre tendría este tipo de movimiento. Pero, la luz fluye y el sustrato también debe hacerlo. Por consiguiente, es razonable asignar una velocidad angular perpendicular al plano de vibración del vector eléctrico que obligará al vector de propagación a cambiar de dirección [Mon19].

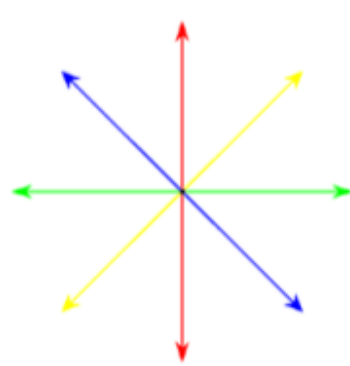

(a)

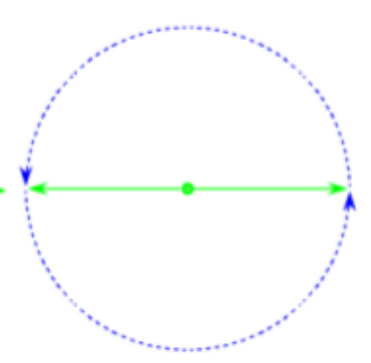

(b)
Figura 4: Diagramas del vector eléctrico para: (a) luz no polarizada y (b) luz polarizada linealmente. La circunferencia indica el ciclo del vector eléctrico asociado a su vibración armónica y el punto central indica la dirección del vector propagación saliendo perpendicularmente del plano del papel.

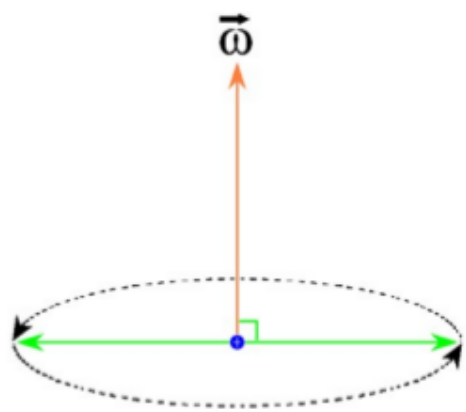

Figura 5: Ciclo de oscilación del vector eléctrico en un plano. Es razonable asignar una velocidad angular perpendicular al plano de vibración del vector eléctrico. Pero este obligará al vector de propagación a cambiar de dirección

A medida que la onda avanza el vector de propagación cambiará de dirección de acuerdo al sentido de la rotación. El diagrama de la Figura 6 muestra tres desplazamientos sucesivos del vector de propagación. Las flechas discontinuas indican desplazamientos aparentes del vector de propagación cuando los puntos del espacio $P_{1}^{\prime}, P_{2}^{\prime}, P_{3}^{\prime}, \ldots$ permanecen fijos. Las flechas continuas indican desplazamientos reales del vector de propagación cuando los puntos del espacio $P_{1}, P_{2}, P_{3}, \ldots$ giran, vibran y fluyen. Es evidente que debe existir una velocidad angular que obligue al vector de propagación a cambiar de dirección en cada instante [Mon19]. 


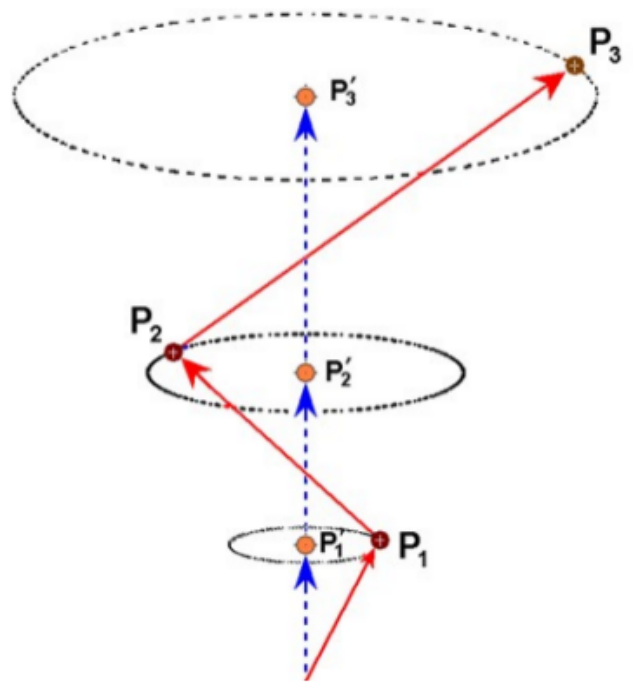

Figura 6: Desplazamientos sucesivos del vector de propagación. Las flechas discontinuas indican los desplazamientos cuando los puntos del espacio vacío $\left(P_{1}^{\prime}, P_{2}^{\prime}, P_{3}^{\prime}, \ldots\right)$ permanecen inmóviles o tienen movimiento de traslación uniforme, y las flechas continuas indican los desplazamientos cuando los puntos del espacio $\left(P_{1}, P_{2}, P_{3}, \ldots\right)$ giran, fluyen y vibran.

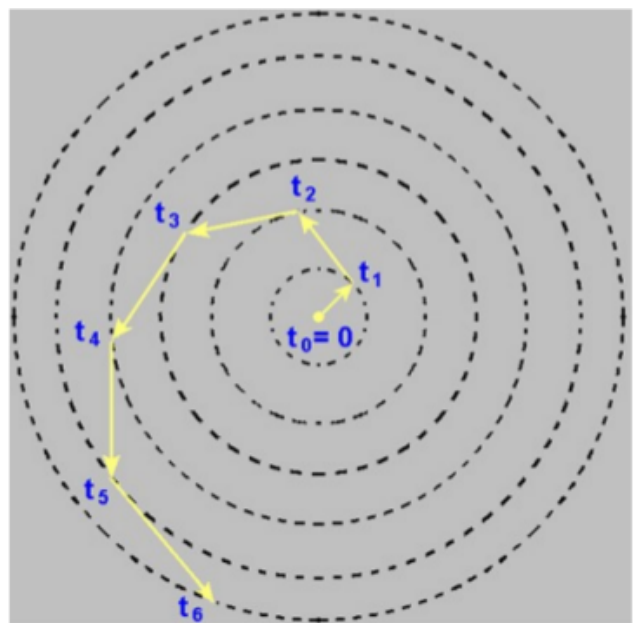

Figura 7: Frentes de onda esféricos (líneas discontinuas) en los instantes $t_{1}, t_{2}, t_{3}, \ldots$ emitidos por una fuente puntual de luz en reposo desde el instante $t_{0}=0$. En un sistema de referencia no inercial los vectores de propagación (flechas continuas) no son perpendiculares a los frentes de onda esféricos sino tangentes. Reduciendo los intervalos de tiempo entre frentes de onda tanto como queramos, el rayo de luz se curvará de acuerdo al sentido de la rotación.

Obsérvese que la velocidad angular asociada tendrá la dirección en que se propagarían los rayos de luz si el sistema de referencia del observador fuese inercial. En consecuencia, si la velocidad angular asociada a un rayo de luz no es nula deberían percibirse vórtices lumino- sos. Sin embargo, no se perciben, lo cual significa que la percepción de la propagación rectilínea de la luz en el espacio libre dependería de un sistema de referencia fundamental: la mente del observador [Mon19].

Para comprender mejor el cambio de dirección del vector de propagación, considérese una fuente puntual de luz y algunos frentes de onda esféricos concéntricos, como muestra la Figura 7. Por simplicidad se puede asumir que en el intervalo de tiempo de $t_{0}=0$ a $t_{1}$ el vector de propagación es perpendicular al frente de onda esférico. En el instante $t_{1}$ el vector de propagación tendrá otra dirección y llegará al siguiente frente de onda esférico en el instante $t_{2}$ en una dirección que no es perpendicular al frente de onda. Análogamente, en el instante $t_{2}$ el vector de propagación volverá a cambiar de dirección llegando al siguiente frente de onda esférico en otra dirección no perpendicular, y así sucesivamente [Mon19].

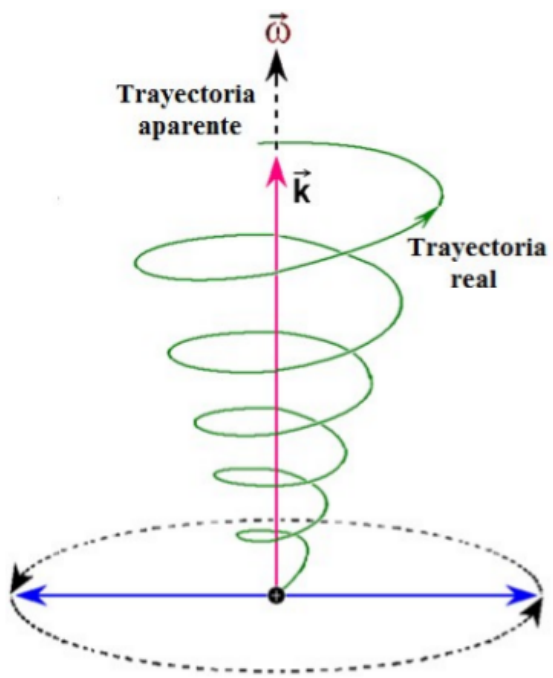

Figura 8: En un sistema inercial el vector de propagación de la onda electromagnética tiene una sola dirección y la trayectoria del rayo de luz es rectilínea. En el sistema no inercial del campo electromagnético el vector de propagación cambia de dirección en cada instante debido a la velocidad angular, y en consecuencia la trayectoria real de un rayo de luz tendría la forma de una espiral cónica.

Obsérvese que a medida que los frentes de onda se expanden el sentido (antihorario) en que cambia de dirección el vector de propagación es de acuerdo a la dirección de la velocidad angular asociada a las vibraciones armónicas del vector eléctrico. Reduciendo los intervalos de tiempo tanto como queramos, el rayo luminoso se curvará, como se muestra en la Figura 8. La trayectoria aparente del rayo de luz se indica con líneas discontinuas y la trayectoria real (que nuestra mente no percibe) se indica con línea continua. Por tanto, la trayectoria real de un rayo de luz procedente de una fuente puntual sería una espiral cónica [Mon19]. 
También, obsérvese que en un sistema de referencia inercial el vector de propagación es perpendicular a cada frente de onda esférico (Figura 1). Por el contrario en un sistema de referencia no inercial el vector de propagación es tangente a cada frente de onda esférico (Figura 7).

\section{Postulado de la cinemática esencial}

El análisis geométrico de la trayectoria de los rayos de luz en el espacio libre perceptible, mostrado en la sección anterior, conduce a postular sobre el comportamiento del sustrato lo siguiente: todos los puntos del espacio vacío de los sistemas naturales giran, fluyen, y vibran con independencia de la consciencia del observador. Considérese un rayo de luz en el espacio libre emitido desde el punto $x=0, y=0, z=0$ en el instante $t=0$. De acuerdo con el postulado anterior, las coordenadas de cada punto del rayo de luz en el vacío en cada instante (el sustrato) se pueden expresar por las ecuaciones paramétricas:

$$
\begin{aligned}
& x(t)=a t \cos \omega t \\
& y(t)=a t \operatorname{sen} \omega t \\
& z(t)=b t
\end{aligned}
$$

donde $a$ y $b$ son constantes positivas. La cantidad $\omega$ se puede interpretar como la magnitud de la velocidad angular asociada a la escala espacial.

Las coordenadas $x(t)$ e $y(t)$ del sustrato oscilan armónicamente con amplitudes crecientes en el tiempo. La coordenada $z(t)$ del sustrato varía linealmente creciente con el tiempo t a medida que la luz se propaga. Es claro que la trayectoria del rayo de luz en el vacío (la espiral cónica) está determinada por el desplazamiento de cada punto del espacio vacío, es decir por el movimiento del sustrato [Mon19]. Derivando las Ecs. (1), (2) y (3) respecto al parámetro $t$, se obtienen las componentes de la velocidad del rayo de luz:

$$
\begin{aligned}
& \frac{d x}{d t}=a \cos \omega t-a t \operatorname{sen} \omega t \\
& \frac{d y}{d t}=a t \operatorname{sen} \omega t+a \omega t \cos \omega t \\
& \frac{d z}{d t}=b
\end{aligned}
$$

Elevando al cuadrado las Ecs. (4),(5) y (6) y sumando se define:

$$
\left(\frac{d x}{d t}\right)^{2}+\left(\frac{d y}{d t}\right)^{2}+\left(\frac{d z}{d t}\right)^{2}=c^{2}(t)
$$

donde $\mathrm{c}(\mathrm{t})$ denota la velocidad variable de la luz dependiente del parámetro del tiempo t. Al sustituir (4),(5) y (6) se tiene:

$$
\begin{gathered}
c^{2}(t)=(\operatorname{acos} \omega t-a \omega t \operatorname{sen} \omega t)^{2}+(\text { asen } \omega t+a \omega t \cos \omega t)^{2}+b^{2} \\
c^{2}(t)=a^{2} \cos ^{2} \omega t+a^{2} \omega^{2} t^{2} \operatorname{sen}^{2} \omega t-2 a^{2} \omega t \cos \omega t \operatorname{sen} \omega t+
\end{gathered}
$$

$$
a^{2} \omega^{2} t^{2} \cos ^{2} \omega t+2 a^{2} \omega t \operatorname{sen} \omega t \cos \omega t+b^{2}
$$

De donde se obtiene:

$$
c^{2}(t)=a^{2}+b^{2}+a^{2} \omega^{2} t^{2}
$$

O también:

$$
c(t)= \pm \sqrt{a^{2}+b^{2}+a^{2} \omega^{2} t^{2}}
$$

Los signos \pm en la Ec.(8) significan que la velocidad de la luz siempre tendría dos direcciones simultáneas posibles, siendo una dirección la opuesta de la otra. Además, obsérvese que si se prescinde de la cinemática esencial del sustrato: $\omega=0$, y la Ec.(8) se reduce a:

$$
c(t)= \pm c
$$

Aquí:

$$
c=\sqrt{a^{2}+b^{2}}=3 \times 10^{8} \mathrm{~m} / \mathrm{s}
$$

Esta es la rapidez de la luz (constante) cuando $\omega=0$. Nótese que las cantidades $a$ y $b$ tienen significado de rapidez, siendo:

$$
a^{2}+b^{2}=c^{2}
$$

De donde necesariamente: $a<c$ y $b<c$. Por consiguiente, sustituyendo (11) en (8) se escribe

$$
c_{\omega}(t)= \pm \sqrt{c^{2}+a^{2} \omega^{2} t^{2}}
$$

Aquí el subíndice $\omega$ indica que la velocidad de la luz depende de la magnitud de la velocidad angular local asociada a la escala espacial.

Obsérvese que la Ec.(11) se puede interpretar geométricamente asociando vectores a las cantidades $a, b$ y $c$, tal como se muestra en la Figura 9. Si $\theta$ es el ángulo que forma el vector asociado $a c$ con el eje de rotación local, es claro que se verifican las relaciones: $a=c \operatorname{sen} \theta$ y $b=\cos \theta$. Entonces las cantidades $a$ y $b$ son las componentes de la velocidad de la luz constante $c$. Además, las cantidades $a$ y $b$ tienen significado de velocidad transversal y velocidad longitudinal respectivamente con respecto al eje de rotación local. También obsérvese que la componente $a$, es paralela a la sección transversal de la espiral cónica y la componente $b$, es perpendicular a ella. 


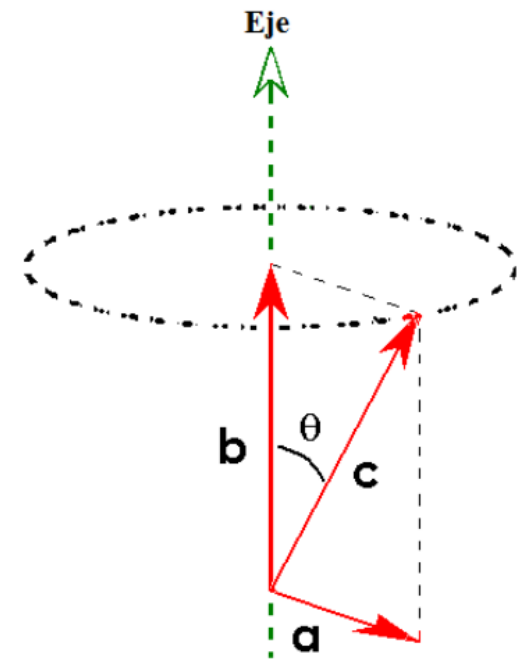

Figura 9: La velocidad constante de la luz $c$, tendría dos componentes respecto a un eje de rotación local: la velocidad transversal $a$ y la velocidad longitudinal $b$.

\section{Criterio del régimen de Planck}

Es claro que en la Ec. (11) no se conocen las cantidades $a$ y $b$. Para determinarlas, considérese la sección transversal de la espiral cónica descrita por el rayo de luz. Elevando al cuadrado las Ecs. (1) y (2), y luego sumándolas, se obtiene el radio de la sección de la espiral cónica:

$$
r(t)=a t
$$

Esta cantidad indica la extensión espacial ocupada por los rayos de luz en cada instante de tiempo $t$. Puesto que el rayo luz debe curvarse en torno a al eje de rotación local, entonces el enrollamiento de todos los rayos que componen un haz de luz determinarían la escala espacial [Mon19].

Así, para determinar las cantidades a y b es necesario introducir un criterio razonable y coherente como sigue: el tamaño y la evolución temporal de un sistema natural sólo tiene sentido a partir de la longitud de Planck y a partir del tiempo de Planck respectivamente [Mon19]. Esto significa que:

$$
r \geq \lambda_{p} ; t \geq t_{p}
$$

donde $\lambda_{p}$ y $t_{p}$ denotan la longitud y el tiempo de Planck respectivamente.

Las condiciones (14) pueden denominarse criterio del régimen de Planck. Por consiguiente, todas las ecuaciones anteriores y las subsiguientes serán válidas siempre que se cumplan las condiciones (14). Evaluando la Ec.(13) en el régimen de Planck se tiene:

$$
r\left(t_{p}\right)=a t_{p}=\lambda_{p}
$$

De donde:

$$
a=\frac{\lambda_{p}}{t_{p}}=\frac{10^{-35}}{10^{-43}}=10^{8} \mathrm{~m} / \mathrm{s}
$$

O también:

$$
a=\frac{1}{3} c
$$

Reemplazando (17) en la Ec.(11) se obtiene:

$$
b=\frac{2 \sqrt{2}}{3} c
$$

Cuando se reemplaza (17) en la Ec.(12), la expresión para la velocidad variable de la luz en el vacío está dada por:

$$
c_{\omega}(t)= \pm c \sqrt{1+(\omega t / 3)^{2}}
$$

donde $t \geq t_{p}$ y $\omega$ es la magnitud de la velocidad angular local asociada a la escala espacial en consideración.

Obsérvese que en la ecuación de la velocidad variable de la luz (19) la magnitud de la velocidad angular local $\omega$ aún no está determinada. Sin embargo, es claro que para una velocidad angular dada de magnitud $\omega$ la velocidad de la luz es creciente en el curso del tiempo. Además, obsérvese que lo signos \pm indican dos posibles direcciones opuestas de la velocidad de la luz.

Por otro lado, utilizando el teorema del binomio se puede desarrollar la fórmula (19) en serie de potencias para expresar la rapidez de la luz en el vacío. El resultado es:

$$
c_{\omega}(t)=c+\frac{1}{2}\left(\frac{\omega t}{3}\right)^{2} c-\frac{1}{8}\left(\frac{\omega t}{3}\right)^{4} c+\ldots
$$

El primer término $c$, de esta serie corresponde a la rapidez constante de la luz en el vacío común, y es una aproximación de orden cero. Esto significa que el postulado de la constancia de la rapidez de la luz de Einstein es solamente una aproximación de orden cero. Por consiguiente, la rapidez de la luz en el vacío sólo sería constante cuando $\omega=0$, es decir cuando la luz se propaga en línea recta.

\section{El modelo del cuanto vectorial}

Cuando se reemplaza (17) en la Ec.(13), el tiempo de circulación del rayo de luz, en que ocupa la extensión espacial de radio $r>\lambda_{p}$, es:

$$
t=\frac{3 r}{c}
$$

Además, asumiendo que el tiempo correspondiente al ciclo de circulación del rayo de luz es igual al tiempo en que cada punto del espacio vacío (el sustrato) realiza un 
ciclo en un plano paralelo a la sección de la espiral cónica, se cumple:

$$
t=\frac{2 \pi}{\omega}
$$

Igualando las Ecs. (21) y (22) se obtiene la expresión:

$$
\omega=\frac{2 \pi c}{3 r}
$$

La Ec.(23) expresa la magnitud de la velocidad angular $\omega$, la cual determina la dirección de circulación de los rayos de luz en cada ciclo de $2 \pi$ rad. Y como es cuantificable a toda escala espacial, tal que $r \geq \lambda_{p}$, se denominará cuanto vectorial local. También, la cantidad r, la cual indica el radio de la extensión espacial ocupada por los rayos de luz, se le puede denominar radio de acción del cuanto vectorial.

El cuanto vectorial local es un modelo simple el cual permite explicar la dirección de la circulación de los rayos de luz. Obsérvese que la magnitud del cuanto vectorial $\omega$ es inversamente proporcional al radio de la extensión espacial $r$. Esto significa que a medida que aumenta el radio de la extensión espacial en cada instante de tiempo $t$, disminuye la magnitud del cuanto vectorial. En la Figura 10 se muestra la representación geométrica del cuanto vectorial local y su radio de acción $r(t)$ desde $t=0$ hasta el instante $t$.

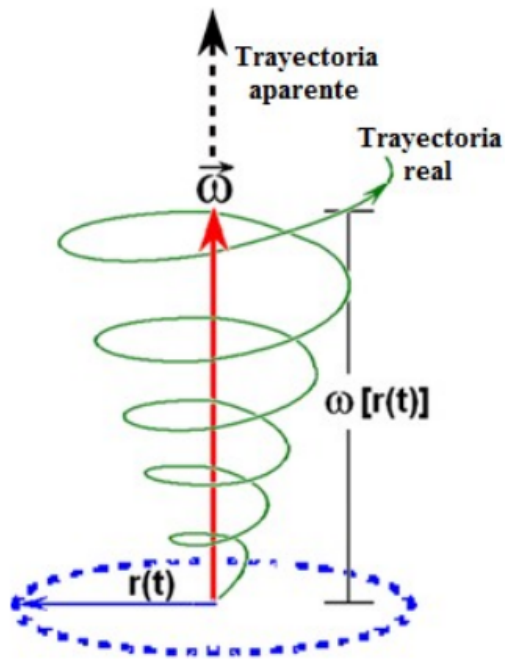

Figura 10: Representación geométrica del cuanto vectorial local. Su radio de acción hasta el instante t está indicado por la circunferencia.
La relación entre los cuantos vectoriales y sus radios de acción se puede interpretar geométricamente como se muestra en la Figura 11. A medida que aumenta el radio de la extensión espacial la magnitud del cuanto vectorial asociado disminuye progresivamente de acuerdo a la Ec.(23). Obsérvese que los cuantos vectoriales $\vec{\omega}_{1}, \vec{\omega}_{2}, \vec{\omega}_{3} \ldots$ pueden ser representativos de las regiones (esferas) espaciales de radios $r_{1}, r_{2}, r_{3}, \ldots$ respectivamente, siendo evidentemente $\omega_{1}>\omega_{2}>\omega_{3}, \ldots$

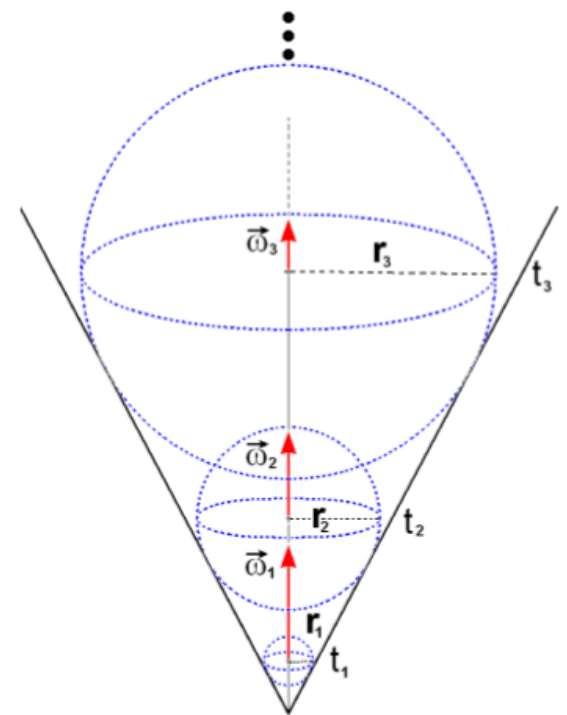

Figura 11: Distribución simple de los cuantos vectoriales a través de extensiones espaciales definidas por esferas. A medida que aumenta el radio de la extensión espacial la magnitud de los cuantos vectoriales representativos disminuye progresivamente

\section{Análisis de la velocidad variable de la luz}

Es de interés comprender la variación de la velocidad de la luz con el tiempo en el espacio vacío de los sistemas naturales para una magnitud dada del cuanto vectorial local en las regiones espaciales típicas de algunos sistemas físicos. En la Tabla 1 se muestran los órdenes de magnitud de los radios de las extensiones espaciales $r$ que son comparables con el tamaño de algunos sistemas físicos. También, se muestran los correspondientes órdenes de magnitud de los cuantos vectoriales $(\omega / 2 \pi)$ representativos de las regiones espaciales. Además, se evalúa la rapidez de la luz en el espacio vacío para $t=1 \mathrm{~s}$. 


\begin{tabular}{lccc}
\hline Sistema & $\mathrm{r}(\mathrm{m})$ & $\frac{\omega}{2 \pi}(\mathrm{Hz})$ & $c_{\omega}(\mathrm{t}=1 \mathrm{~s})(\mathrm{m} / \mathrm{s})$ \\
\hline Núcleo atómico & $10^{-15}$ & $10^{23}$ & $10^{23} \mathrm{c}$ \\
Átomo & $10^{-10}$ & $10^{18}$ & $10^{18} \mathrm{c}$ \\
Humano & 1 & $10^{8}$ & $10^{8} \mathrm{c}$ \\
Tierra-Luna & $10^{9}$ & $10^{-1}$ & $c$ \\
Solar & $10^{11}$ & $10^{-3}$ & $c$ \\
Vía láctea & $10^{20}$ & $10^{-12}$ & $c$ \\
\hline
\end{tabular}

Tabla 1: Órdenes de magnitud de los radios de las extensiones espaciales para evaluar la rapidez de la luz

Los valores de los cuantos vectoriales representativos $(\omega)$ que se muestran en la Tabla 1 confirman su comportamiento decreciente de acuerdo a la Ec.(23). Es de particular importancia interpretar el valor del cuanto vectorial correspondiente a la escala humana: $\omega_{H} \sim 10^{8} \mathrm{rad} / \mathrm{s}$.

También, en la Tabla 1 se muestran los valores que se obtienen para la rapidez de la luz cuando esta se evalúa en el instante $t=1 \mathrm{~s}$. Obsérvese que para regiones del espacio comparables al tamaño de un núcleo atómico es $\sim 10^{23} c$; en la escala atómica es $\sim 10^{18} c$ y en la escala humana es $\sim 10^{8} \mathrm{c}$. La rapidez de la luz es aún mayor cuando la se evalúa para $t>>1 \mathrm{~s}$, porque según la Ec.(19), e inclusive a gran escala, se obtiene $\left|c_{\omega}(t)\right| \gg c$.

Los valores de $c_{\omega}(t)$ pueden incrementarse con el tiempo en cada nivel de estructuras físicas. La razón es que a cada nivel de estructuras físicas se le puede asociar un cuanto vectorial representativo el cual puede fijarse durante su periodo de acción sobre los rayos de luz, y como dicho intervalo de tiempo tiene infinitos valores de $t$, entonces la velocidad de la luz puede incrementarse indefinidamente en dicho intervalo de tiempo [Mon19].

En la Figura 12 se muestra la distribución de los cuantos vectoriales representativos en una línea de tiempo correspondiente a escalas espaciales comparables a las escalas nuclear $\left(\bar{\omega}_{N}\right)$, atómica $\left(\bar{\omega}_{A}\right)$, humana $\left(\bar{\omega}_{H}\right)$, solar $\left(\bar{\omega}_{H}\right)$ y de la vía láctea $\left(\bar{\omega}_{V L}\right)$. Es conveniente definir la cantidad $\bar{\omega}=\frac{\omega}{2 \pi}=a / r,(a=c / 3)$ como la magnitud del cuanto vectorial natural.

Utilizando órdenes de magnitud se puede demostrar fácilmente que en cada ciclo de circulación de los rayos de luz, que equivale al ciclo de operación del cuanto vectorial, y en cualquier escala de estructuras físicas, se cumple: $\bar{\omega} t=1$. Obsérvese que fijando un cuanto vectorial representativo durante el intervalo tiempo en que opera, la velocidad de la luz puede incrementarse indefinidamente con el tiempo.

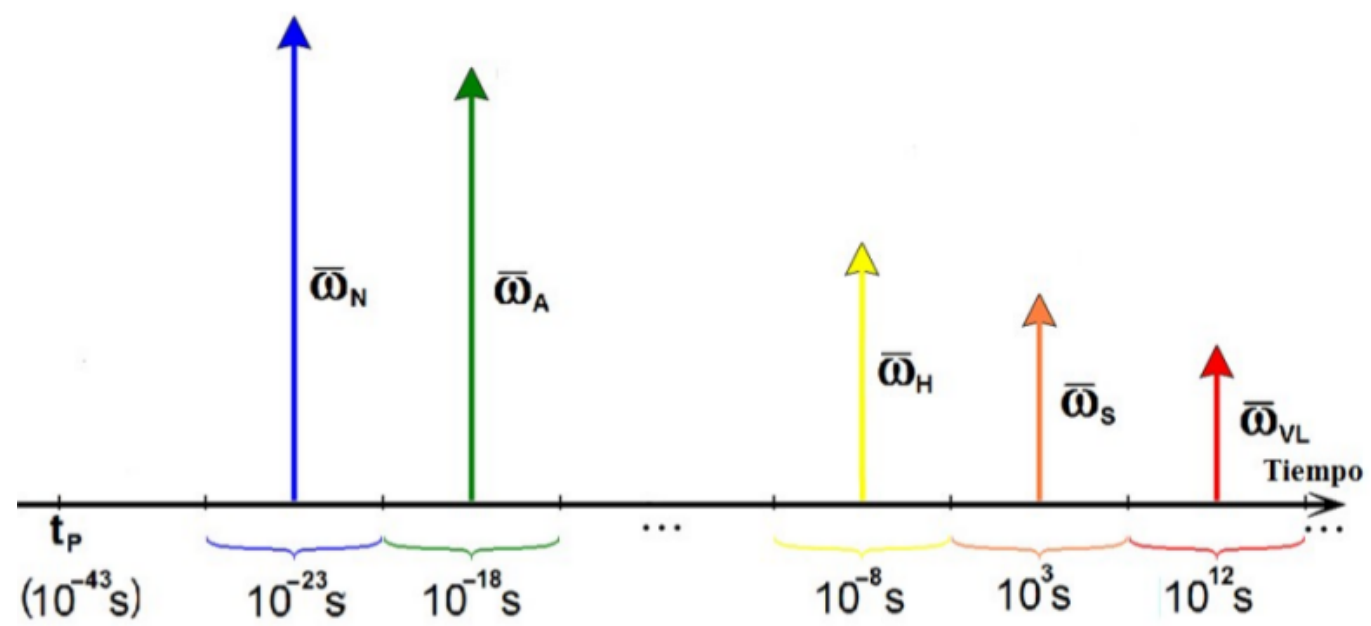

Figura 12: Distribución de algunos cuantos vectoriales representativos. Cada cuanto vectorial es representativo de la escala de estructuras físicas en consideración y tiene un periodo de acción típico sobre los rayos de luz. 
En la Figura 13 se muestra el comportamiento de la velocidad variable de la luz para tres cuantos vectoriales de magnitudes $\omega_{1}, \omega_{2}, \omega_{3}$, tales que $\omega_{1}>\omega_{2}>\omega_{3}$. Se observa que la velocidad variable de la luz se reduce asintóticamente a los valores $c_{0}(t)= \pm c$, para $\omega=0$. Así, para cada cuanto vectorial local dado, la rapidez de la luz en el espacio vacío de los sistemas naturales se incrementa de modo continuo con el tiempo, tal como lo prescribe la Ec.(19). Para un mismo intervalo de tiempo la luz es mucho más rápida en el nivel microscópico que en el nivel macroscópico, porque según la Ec.(23) la magnitud del cuanto vectorial en el nivel microscópico es mucho mayor que el nivel macroscópico [Mon19].

También, en la Figura 14 se observa que la velocidad variable de la luz tiene en todo instante dos direcciones opuestas, siempre que:

$$
\left|c_{\omega}(t)\right|>c
$$

A estas dos direcciones le corresponden respectivamente dos cuantos vectoriales locales exactamente opuestos $\pm \vec{\omega}$. Además, la velocidad variable de la luz es simétrica con respecto al rango de velocidades:

$$
-c \leq v \leq c
$$

Este rango, que se muestra en la región sombreada de la Figura 14, corresponde al estudio de la propagación rectilínea de la luz en un sistema aparentemente inercial, es decir corresponde al nivel de descripción de la Física convencional [Mon19]. Por consiguiente, la condición (24) establece un nuevo nivel de descripción de la Física.

En la Figura 14 se muestra la gráfica del cuanto vectorial local $(\omega)$ en función de su radio de acción $(r)$ a partir de la escala de Planck. A cualquier escala espacial, tal que $r \geq \lambda_{p}$, siempre existirán dos cuantos vectoriales opuestos $\pm \vec{\omega}$. Nótese que no hay comportamiento asintótico cuando el radio de acción $r \rightarrow \lambda_{p}$, ya que $\omega$ tiene un valor finito dado por la Ec.(23):

$$
\omega_{p}=\frac{2 \pi c}{3 \lambda_{p}} \sim 10^{-42} \mathrm{~Hz}
$$

También, de la Figura 14 se observa que cuando $r \rightarrow \infty, \omega \rightarrow 0$. Esto significa que a gran escala espacial los cuantos vectoriales locales son de magnitud despreciable, el universo se comportaría como un sistema inercial y la luz se propagaría en línea recta. En caso contrario, si el radio de acción del cuanto vectorial es finito no se debería despreciar su efecto sobre los rayos de luz [Mon19].

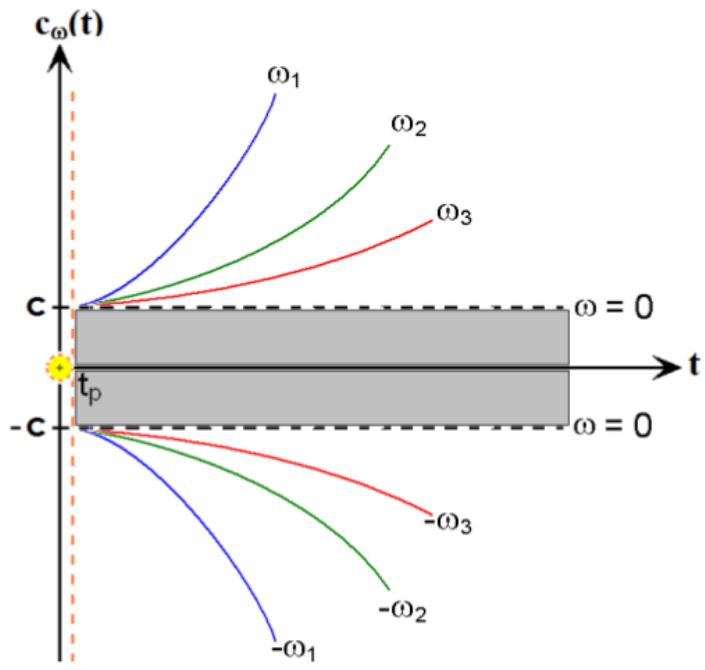

Figura 13: Comportamiento de la velocidad variable de la luz $c_{\omega}(t)$ con el tiempo $\left(t \geq t_{p}\right)$ para tres cuantos vectoriales $\left(\omega_{1}>\omega_{2}>\omega_{3}\right)$. Nótese que en todo instante hay dos direcciones de propagación opuestas correspondientes a los cuantos vectoriales $\pm \omega$. Hay simetría especular respecto al rango de velocidades entre $\pm c$ (región sombreada). El valor $\omega=0$ corresponde a un sistema inercial donde la rapidez de la luz se reduce al valor constante $c$. Es evidente que la doble dirección de la velocidad de la luz está relacionada con la doble dirección del cuanto vectorial

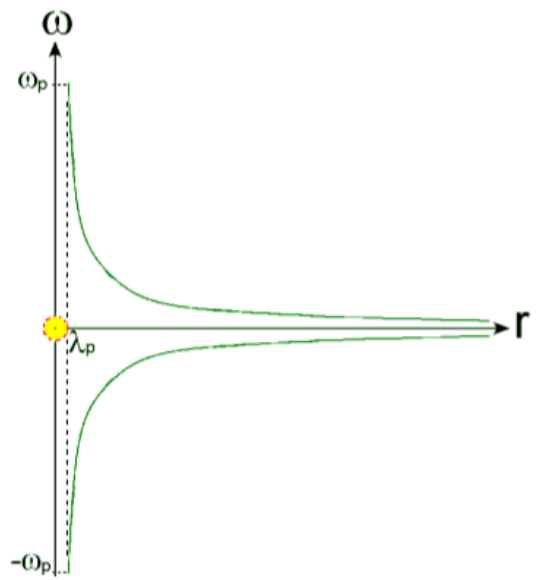

Figura 14: Comportamiento del cuanto vectorial local $\omega$ en función de su radio de acción $r$, siendo $r \geq \lambda_{p}$. Nótese que cuando el radio de acción del cuanto vectorial local es muy grande $(r=\infty)$ el universo a gran escala podría considerarse un sistema inercial $(\omega=0)$ y los rayos de luz se pueden describir en línea recta.

Teóricamente los cuantos vectoriales opuestos que se muestran en las figuras 13 y 14 se equilibran exactamente a toda escala espacial. Pero empíricamente no se equilibrarían exactamente. Por consiguiente es razonable asu- 
mir que los cuantos vectoriales denotados por $\vec{\omega}$ y $\vec{\omega}^{\prime}$ son opuestos pero estarían en cuasiequilibrio. Esto significa que sus magnitudes tendrían fluctuaciones intrínsecas $\Delta \omega$ y $\Delta \omega^{\prime}$, las cuales estarían correlacionadas con las frecuencias de vibración características que se detectan en los sistemas naturales. Por consiguiente, una condición necesaria de cuasiequilibrio para que los rayos de luz se perciban en línea recta es:

$$
\vec{\omega}+\vec{\omega}^{\prime} \approx \overrightarrow{0}
$$

La implicación inmediata de la condición (27) es que $\Delta \omega \ll \omega$ y $\Delta \omega^{\prime} \ll \omega^{\prime}$. Los casos $\Delta \omega \sim \omega$ y $\Delta \omega^{\prime} \sim \omega^{\prime}$ corresponderían a estados de desequilibrio del sistema.

\section{Conclusiones}

En un sistema inercial el vector de propagación de la onda electromagnética en el espacio libre perceptible tiene una sola dirección y la trayectoria del rayo de luz es rectilínea. Considerando el campo electromagnético como sistema no inercial, el vector de propagación cambia de dirección en cada instante debido a la velocidad angular asociada al plano de vibración del campo eléctrico/magnético, y en consecuencia la trayectoria real de un rayo de luz tendría la forma de una espiral cónica.

El análisis geométrico de la trayectoria de los rayos de luz en el espacio libre conduce a una formulación simple de un conjunto de ecuaciones paramétricas que representarían la cinemática esencial de los puntos del espacio vacío de los sistemas naturales, es decir del sustrato esencial. A partir de estas ecuaciones se deduce la ecuación que describe la variación de la velocidad de la luz con el tiempo en el espacio vacío de los sistemas naturales.

La velocidad de la luz sólo sería constante en aproximación de orden cero, es decir sólo en un sistema de referencia inercial hipotético, donde se prescinde de la velocidad angular asociada a los rayos de luz.

El modelo del cuanto vectorial local permite determinar la dirección de circulación de los rayos de luz en cada ciclo de $2 \pi$ rad. Es cuantificable a toda escala espacial mediante su radio de acción y modula la trayectoria de los rayos de luz el espacio vacío de los sistemas naturales a toda escala espacial.

La doble dirección de la velocidad de la luz estaría correlacionada con la doble dirección del cuanto vectorial. La simetría especular de la velocidad variable de la luz significa que si una de las direcciones del cuanto vectorial corresponde a la propagación de la luz en el espacio vacío real de los sistemas naturales, la otra dirección corresponde a la propagación de la luz en el espacio vacío virtual de los sistemas naturales.

La condición de cuasiequilibrio entre los cuantos vectoriales asociados a los espacios real y virtual sería una razón fundamental por la cual se percibe la propagación de la luz en línea recta.

\section{Referencias}

[Ein86] Einstein, A., Infeld L., (1986). La evolución de la Física, Salvat Editores, S.A., Barcelona, España.

[Haw05] Hawking, S., Mlodinow, L., (2005). Brevísima Historia del Tiempo, Ed. Crítica, S.L, Barcelona, España.

[Hec77] Hecht, E. y Zajac, A. (1977). Óptica, Fondo Educativo Interamericano, S.A, Estados Unidos.

[Mag06] Magueijo, J. (2006). Más rápido que la velocidad de la luz, Ed. Fondo de Cultura Económica S.A, Argentina.

[Meh08] Mehdipour, S., Nozari, K. y Sadatian, D. (2008). Análisis fraccional de la propagación del paquete de onda y algunos aspectos de la velocidad variable de la luz con el principio de incertidumbre generalizado, Fractals 16, 33 doi: 10.1142/s0218348x0800379x, Inglaterra.

[Mof02] Moffat, J. (2002). Variable Speed of Light
Cosmology and Second Law of Thermodynamics, cds.cem.ch, Canadá.

[Mon18] Monroy, O. (2018). Análisis de un sistema de referencia inercial, Revista de Investigación de Física, UNMSM, Vol 21, Nº1, Perú.

[Mon19] Monroy, O. (2019). Variación de la velocidad de la luz en el vacío, Enseñanza y Aprendizaje, Editorial Académica Española, España.

[Pet88] Petit, J. (1988). Modelo cosmológico con velocidad variable de la luz: la interpretación del corrimiento hacia el rojo, Mod. Phys. Lett. A 3(18): 1733-1744, Francia.

[Rac09] Racker, J., Sistema, P., Vucetich, H. (2009). Thermodynamics in variable speed of light theories, Physical Review D 80, Estados Unidos.

[Ros66] Rossi, B. (1966). Fundamentos de Óptica, Editorial Reverté, S.A, España.

[Ser06] Serway, R., Moses, C. y Moyer, C. (2006). Fisica Moderna, Thomson Ed., México 